\title{
Methine-Quinoidal Fragment Induces Significant Bathochromic Shifts in Organic Dyes
}

Tianruo Shen, ${ }^{\dagger}$ Ying Gao, ${ }^{\dagger, \dagger}$ Chao Wang, ${ }^{\dagger, \S}$ Zhaochao Xu ${ }^{\S}$ and Xiaogang Liu ${ }^{*, \dagger}$

$\uparrow$ Fluorescence Research Group, Singapore University of Technology and Design, 8 Somapah Road, Singapore 487372, Singapore.

\$ Jilin Engineering Normal University, 3050 Kaixuan Road, Changchun 130052, China.

$\S$ CAS Key Laboratory of Separation Science for Analytical Chemistry, Dalian Institute of Chemical Physics, Chinese Academy of Sciences, 457 Zhongshan Road, Dalian 116023, China. 


\section{Contents}

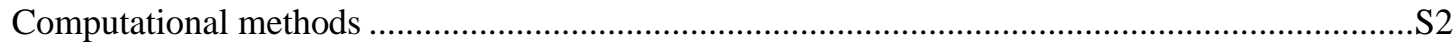

Comparison of functionals and solvent formalisms in calculating $\lambda_{\text {abs }}$ of $\mathbf{1}$ and $\mathbf{2} \ldots \ldots \ldots \ldots \ldots \ldots \ldots \ldots \ldots . . . . . . . . . . . .33$

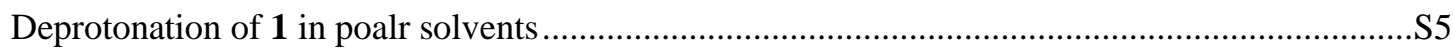

Molecular designs with quinoidal structures by exchanging the sequence of a conjugated ring and

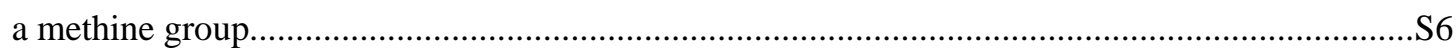

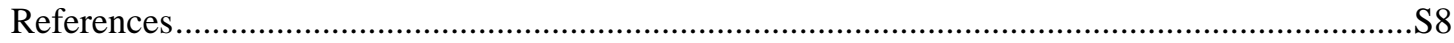




\section{Computational methods}

The molecular structures of $\mathbf{1}$ and $\mathbf{2}$ in the ground state were optimized using density functional theory (DFT) with various density functionals listed below. All optimized molecular structures were confirmed at the local minima on the potential energy surfaces through frequency calculations with various density functionals listed below. Based on these optimized structures, peak UV-vis absorption wavelengths $\left(\lambda_{\text {abs }}\right)$ of $\mathbf{1}$ and $\mathbf{2}$ were calculated using time-dependent density functional theory (TD-DFT). We considered seven functionals, including PBE, B3LYP, PBE0, BMK, M06-2X, CAM-B3LYP, and $\omega$ B97XD combing with def2-SVP ${ }^{1}$ basis set (unless stated otherwise) with reference to experimental data.

We accounted solvation effects using SMD model, with linear-response (LR), corrected linearresponse (cLR) and state-specific (SS) solvent formalisms, in ten solvents with different polarities, including cyclohexane (CYH, dielectric constant $\varepsilon=2.0165$ ), dioxane (Diox, $\varepsilon=2.2099$ ), diethyl ether (Ether, $\varepsilon=4.240$ ), tetrahydrofuran (THF, $\varepsilon=7.4257$ ), 2-propanol (IPA, $\varepsilon=19.264$ ), ethanol $(\mathrm{EtOH}, \varepsilon=24.852)$, methanol $(\mathrm{MeOH}, \varepsilon=32.613)$, acetonitrile $(\mathrm{ACN}, \varepsilon=35.688)$, dimethyl sulfoxide (DMSO, $\varepsilon=46.826)$ and water $(\varepsilon=78.3553)$.

All DFT and TD-DFT calculations were conducted using Gaussian $16 .^{2}$

Multiwfn ${ }^{3}$ and VMD ${ }^{4}$ software were used to construct electrostatic potential (ESP) surfaces, conduct hole-electron analysis, and calculate the corresponding charge transfer distances $\left(d_{\mathrm{CT}}\right)$. 


\section{Comparison of functionals and solvent formalisms in calculating $\lambda_{\text {abs }}$ of 1 and 2}
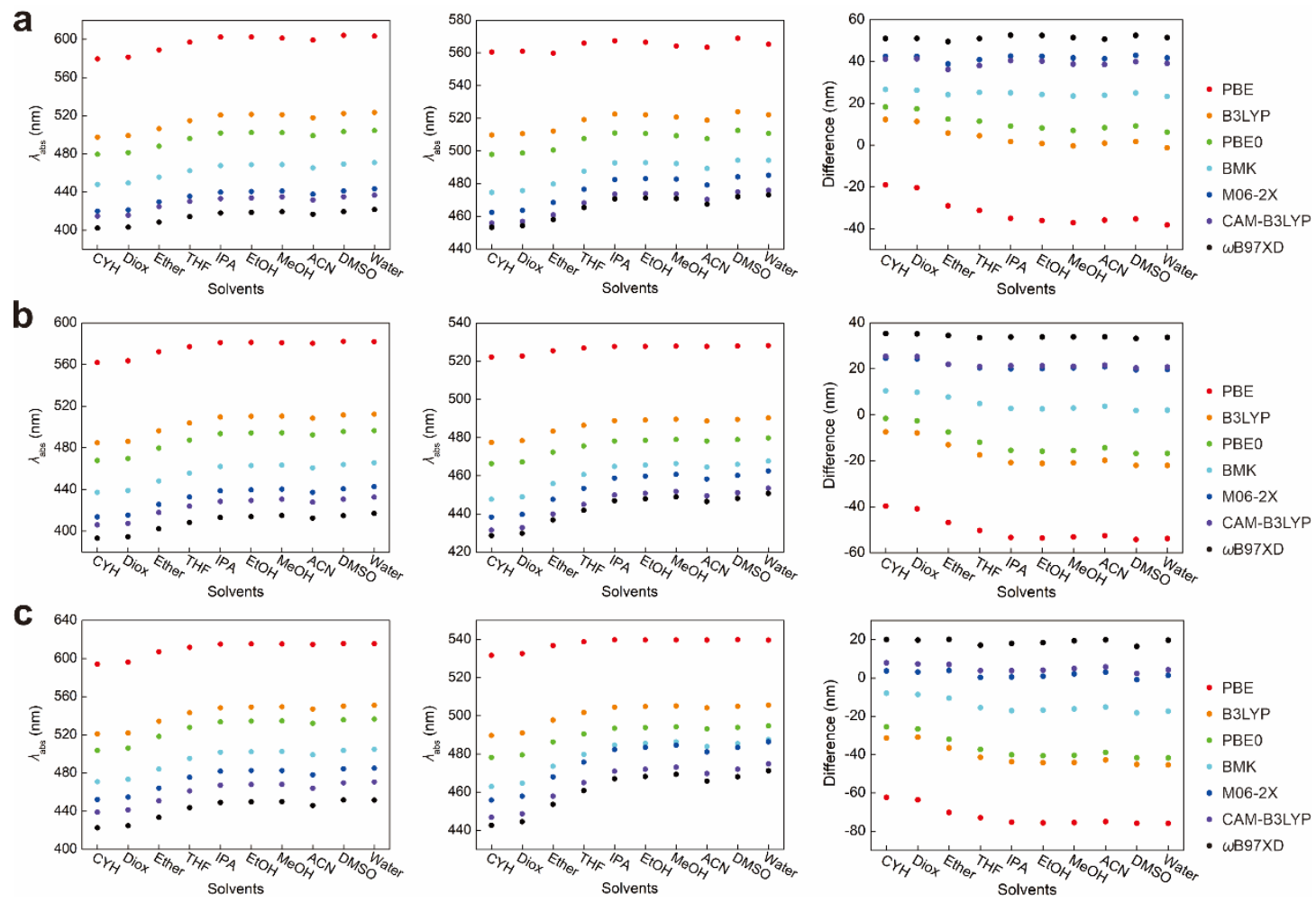

Figure S1. Calculated peak UV-vis absorption wavelengths ( $\lambda_{\text {abs }}, \mathrm{nm}$ ) of 1 (left), 2 (middle) and their difference ( $\lambda_{\text {abs }}$ of $\mathbf{2}-\lambda_{\text {abs }}$ of $\mathbf{1}$; right) in ten solvents using various functionals with (a) linear-response (LR), (b) corrected linear-response (cLR), and (c) state-specific (SS) solvent formalisms. 

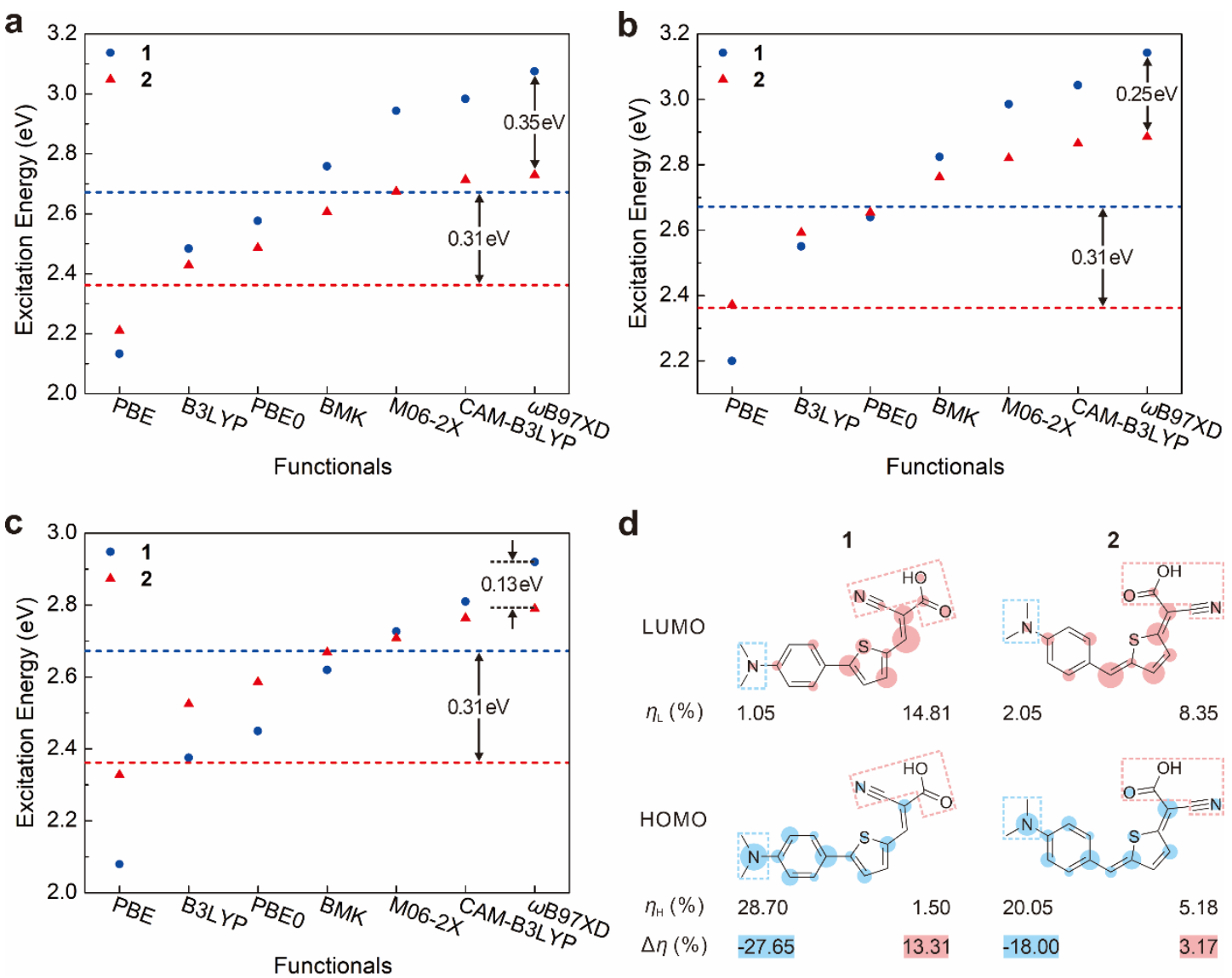

Figure S2. Calculated excitation energies of $\mathbf{1}$ and $\mathbf{2}$ using different functionals in dioxane with (a) linear-response (LR), (b) corrected linear-response (cLR) and (c) state-specific (SS) solvent formalisms; (d) Atomic contributions to the HOMO $\left(\eta_{\mathrm{H}}\right)$ and LUMO $\left(\eta_{\mathrm{L}}\right)$ of $\mathbf{1}$ and $\mathbf{2}$ in dioxane, and the change of atomic contributions upon HOMO-LUMO transition $(\Delta \eta)$.
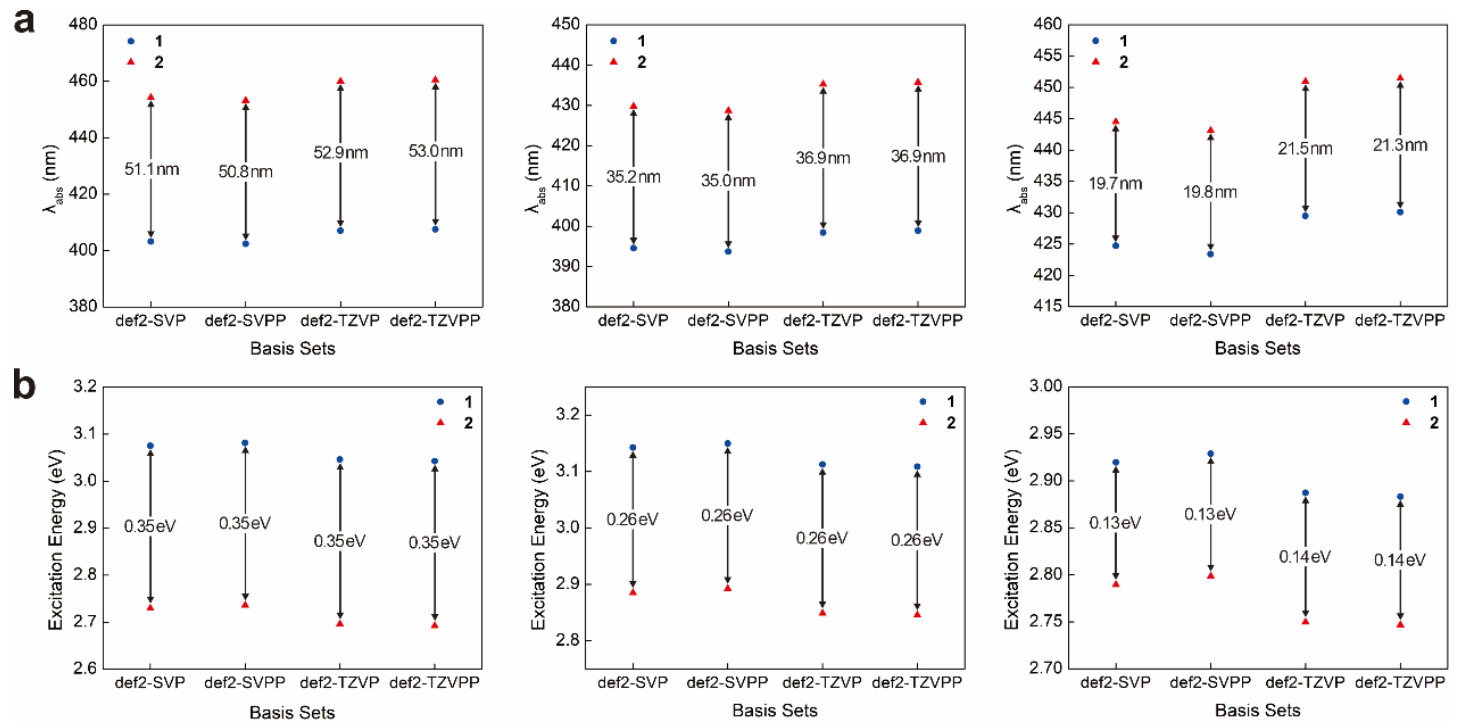

Figure S3. Calculated (a) peak UV-vis absorption wavelengths $\left(\lambda_{\mathrm{abs}}, \mathrm{nm}\right)$ and (b) excitation energies of $\mathbf{1}$ and $\mathbf{2}$ in using $\omega \mathrm{B} 97 \mathrm{XD}$ in combination with four different basis sets with linearresponse (LR, left), corrected linear-response (cLR, middle), and state-specific (SS, right) solvent formalisms. 


\section{Deprotonation of 1 in polar solvents}

Overall, our calculations suggest a positive solvatochromism for both $\mathbf{1}$ and $\mathbf{2}$ (Figure 1). However, experimental data showed that 1 displayed a negative solvatochromism from dioxane to DMSO, in contrast to the positive solvatochromism in $\mathbf{2}$.

Inspired by the hyphosis of Segawa et al. on the deprotonation of $\mathbf{1}$ in polar solvents, ${ }^{5}$ we computed $\lambda_{\text {abs }}$ of both 1 and deprotonated 1 in DMSO (Figure S4a). Our data showed that the deprotonation led to a substantial blue shift in the UV-vis absorption peaks.

We also compared the charge densities of carboxyl (-COOH) groups of both $\mathbf{1}$ and $\mathbf{2}$ in DMSO, to understand the higher tendency of deprotonation in $\mathbf{1}$ (Figure S4b). Our results show that the partial positive charges accumulate in $-\mathrm{COOH}$ group of $\mathbf{1}$, while $\mathbf{2}$ carries a partial negative charge at $-\mathrm{COOH}$ group. Due to the lower charge density on the $-\mathrm{COOH}$ group in $\mathbf{1}$, this compound demonstrates a higher deprotonation tendency. Accordingly, the deprotonation of $\mathbf{1}$ could successfully rationalize the observed experimental data on the spectral shifts of both $\mathbf{1}$ and 2 going from dioxane to DMSO (Figure S4c). For example, the $\lambda_{\text {abs }}$ of 2 shows a redshift from dioxane to DMSO, while 1 appears to possess a negative solvatochromism.
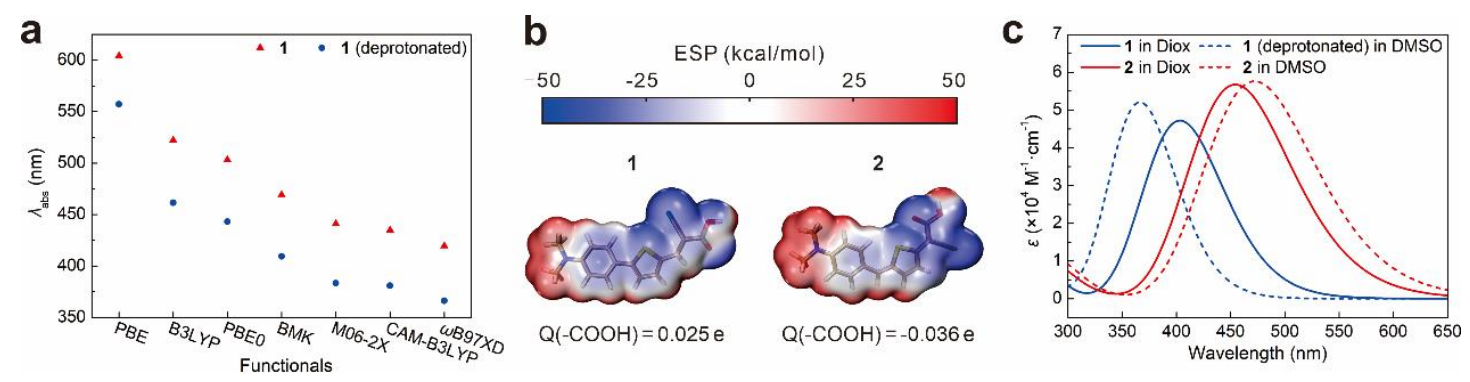

Figure S4. (a) Calculated peak UV-vis absorption wavelengths $\left(\lambda_{\text {abs }}, \mathrm{nm}\right)$ of $\mathbf{1}$ and deprotonated $\mathbf{1}$ in DMSO using different functionals; (b) Electrostatic potential (ESP) surfaces of $\mathbf{1}$ and $\mathbf{2}$ in DMSO, and the partial charge at the carboxyl group ( $Q_{-\mathrm{cooH}}$, e); (c) Calculated UV-vis absorption spectra of $\mathbf{1}$ (or deprotonated $\mathbf{1}$ ) and $\mathbf{2}$ in dioxane and DMSO. 
Molecular designs with quinoidal structures by exchanging the sequence of a conjugated ring and a methine group

a

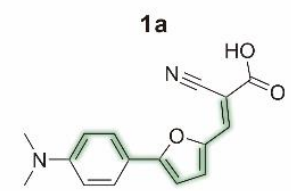

$1 \mathrm{~b}$
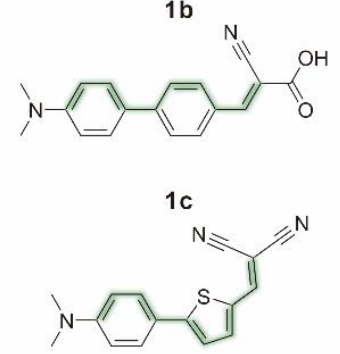

$1 d$
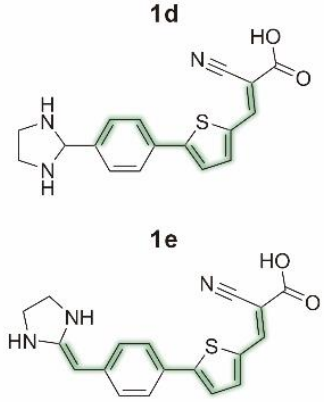

b

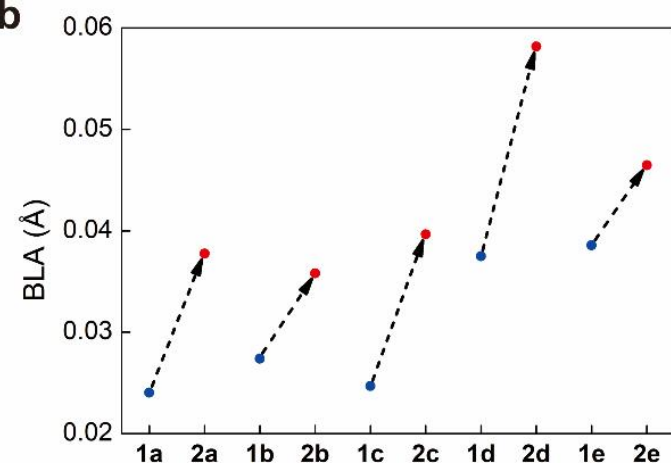

C

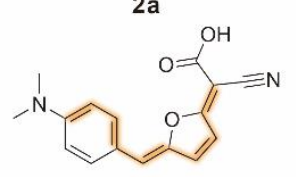

$2 b$
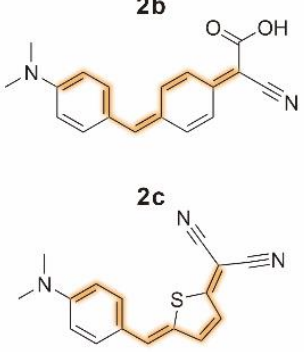

$2 d$

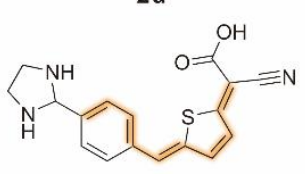

$2 \mathrm{e}$

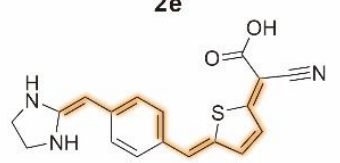

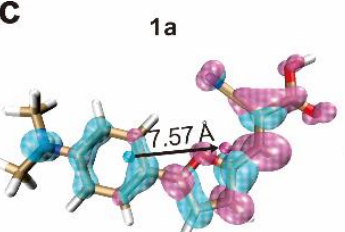

$1 \mathrm{~b}$

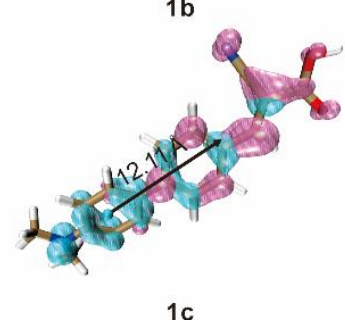

$1 c$

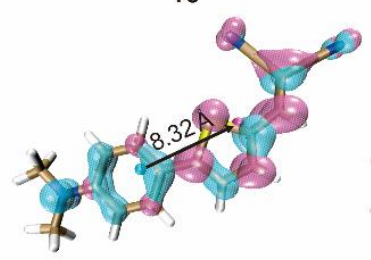

$1 d$

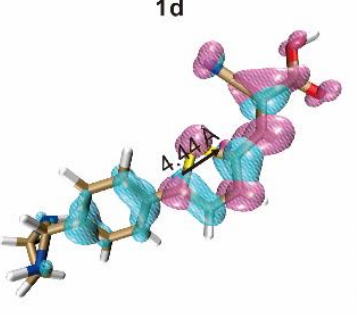

$1 \mathrm{e}$

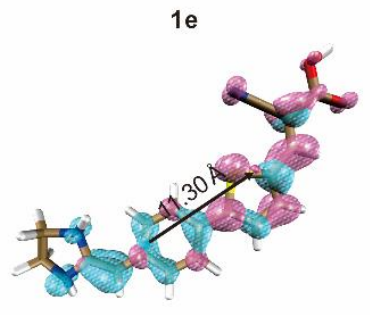

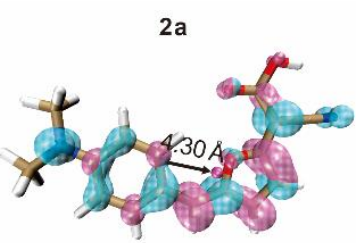

$2 \mathrm{~b}$

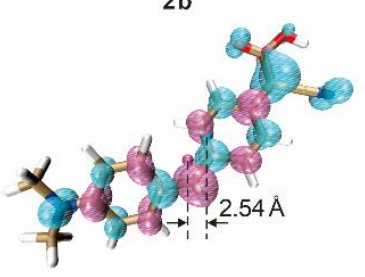

2c

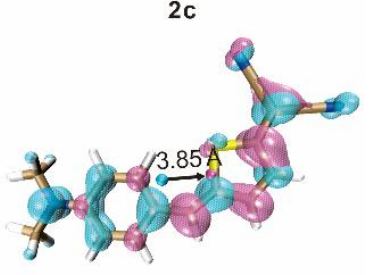

$2 d$

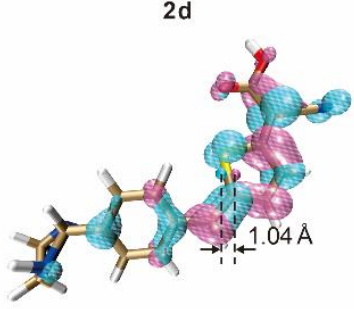

$2 \mathrm{e}$

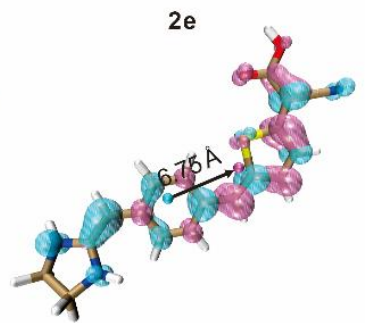

Figure S5. (a) Molecular structures of 1a-1e and 2a-2e; the conjugated paths for calculating bond-length alternation (BLA) values are highlighted in green and orange; (b) Calculated bondlength alternation (BLA) values of 1a-1e and 2a-2e based on the optimized geometries of these compounds in dioxane in the ground state $\left(\mathrm{S}_{0}\right)$; (c) Hole-electron analysis with charge transfer distances of 1a-1e and 2a-2e in dioxane; cyan: hole, pink: electron. 
a
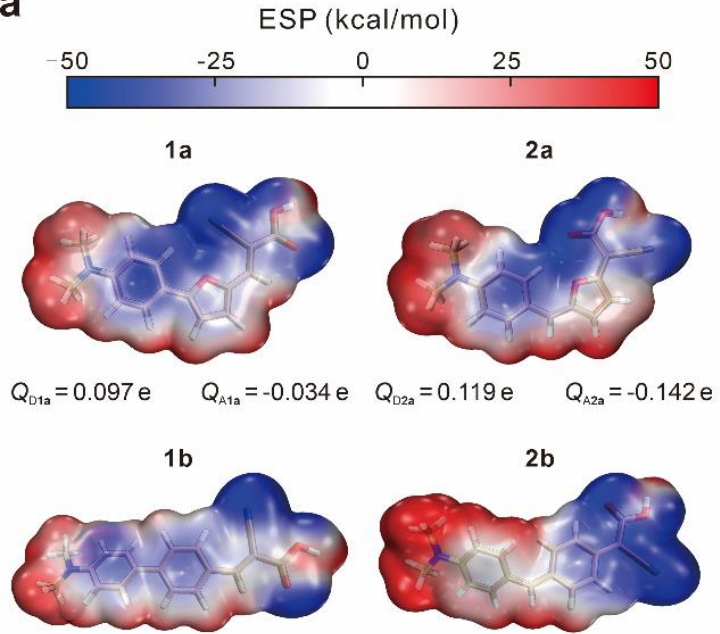

$Q_{D 1 b}=0.080 e$
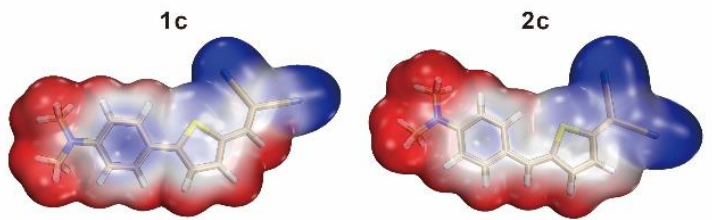

$Q_{D 1 c}=0.102$
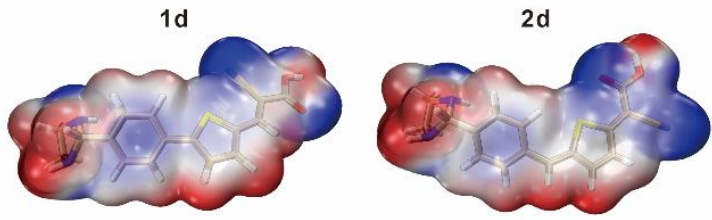

$Q_{D 1 d}=0.130 \mathrm{e}$
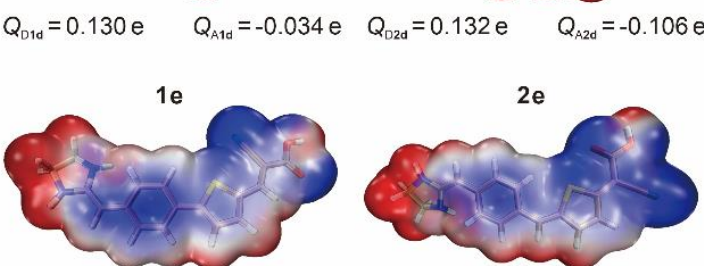

$Q_{D 1 e}=0.162 \mathrm{e}$

b
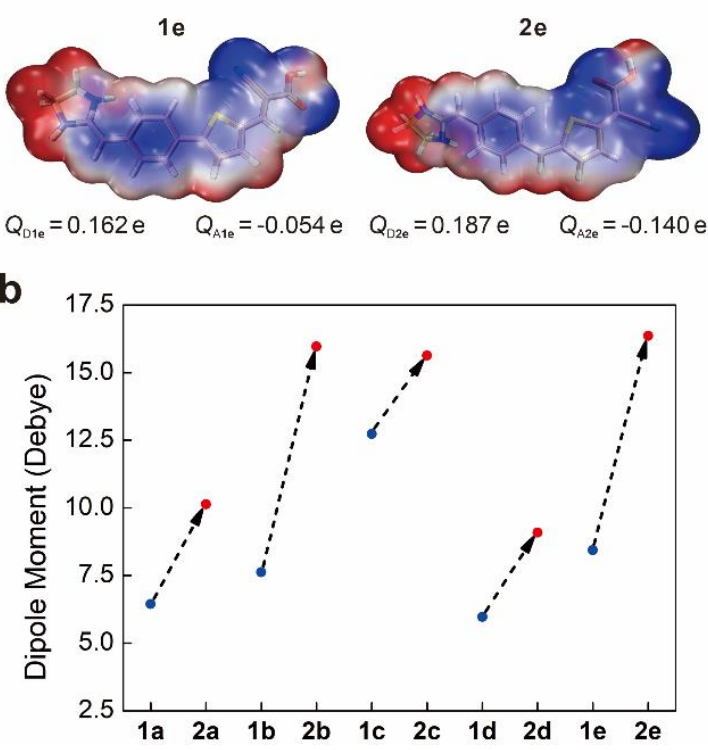

C

LUMO

$\eta_{L}(\%)$

HOMO

$\eta_{H}(\%)$

$\Delta \eta(\%)$

LUMO

$\eta_{\mathrm{L}}(\%)$

$\eta_{L}(\%)$
HOMO

$\eta_{H}(\%)$

$\Delta \eta(\%)$

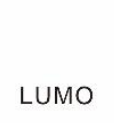

LUMO

$\eta_{L}(\%)$

HOMO

$\eta_{\mathrm{H}}(\%)$

$\Delta \eta(\%)$

LUMO
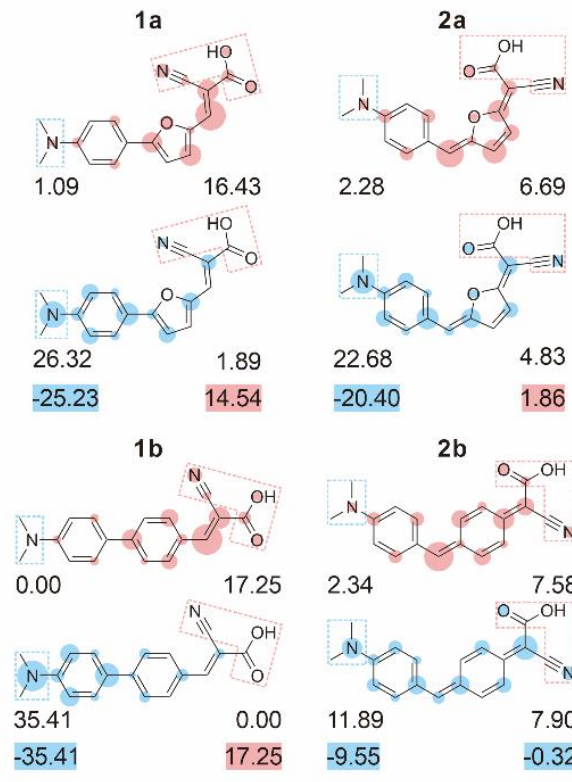

26.32

$-25.23$

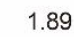

14.54

$1 b$
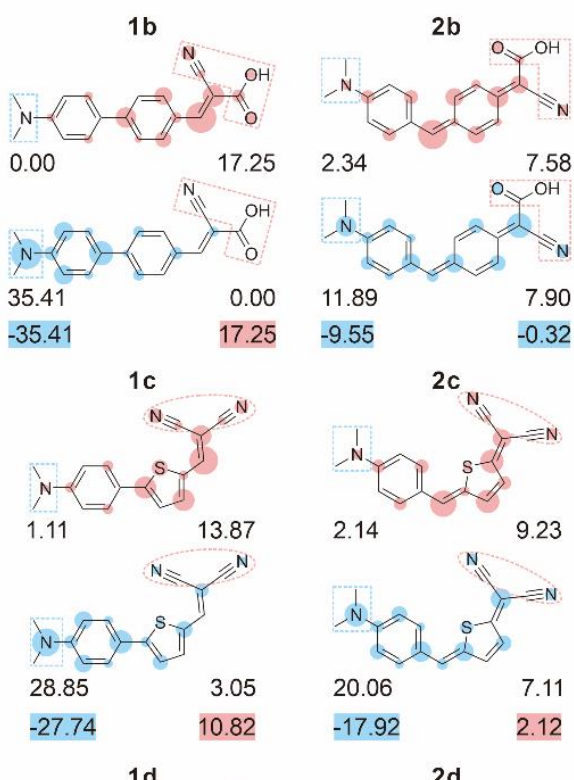

$-9.55$
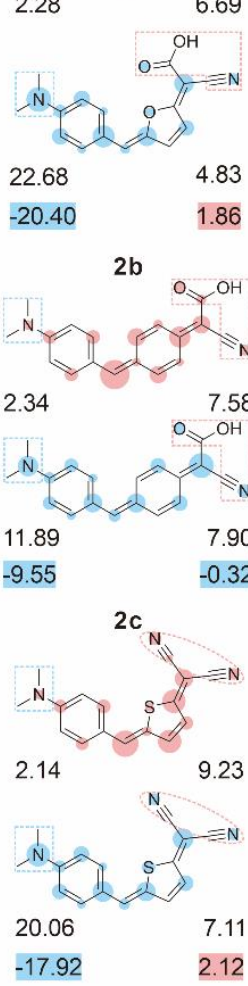

2.12
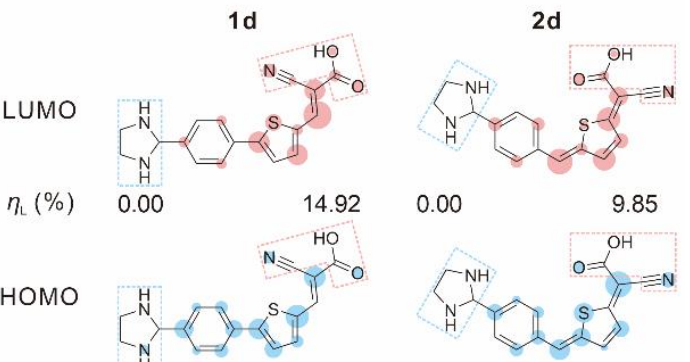

$\eta_{\mathrm{H}}(\%)$

$\Delta \eta(\%) \quad-2.05$

6.01

0.00

9.05

8.91

$0.00 \quad 0.80$

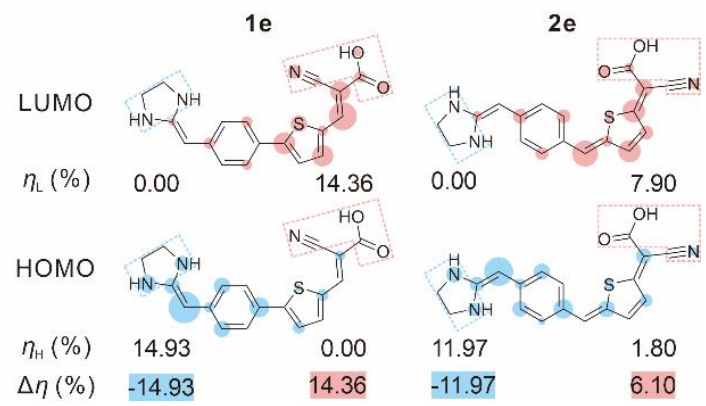

Figure S6. (a) Electrostatic potential (ESP) surfaces of 1a-1e and 2a-2e in dioxane, and the partial charge at donors $\left(Q_{\mathrm{D} 1 \mathrm{x}}, \mathrm{e}\right)$ and acceptors $\left(Q_{\mathrm{A} 1 \mathrm{x}}, \mathrm{e}\right)$; (b) Calculated dipole moments of 1a1e and 2a-2e in dioxane at the ground state $\left(\mathrm{S}_{0}\right)$. (d) Atomic contributions to HOMO $\left(\eta_{\mathrm{H}}\right)$ and LUMO $\left(\eta_{\mathrm{L}}\right)$ and the corresponding changes $(\Delta \eta)$ upon HOMO-LUMO transition of 1a-1e and 2a-2e in dioxane. 


\section{References}

1. Weigend, F.; Ahlrichs, R. Balanced Basis Sets of Split Valence, Triple Zeta Valence and Quadruple Zeta Valence Quality for H to Rn: Design and Assessment of Accuracy. Phys. Chem. Chem. Phys. 2005, 7, 3297-3305.

2. Frisch, M.; Trucks, G.; Schlegel, H.; Scuseria, G.; Robb, M.; Cheeseman, J.; Scalmani, G.; Barone, V.; Petersson, G.; Nakatsuji, H.; et al. Gaussian 16, Gaussian, Inc., Wallingford CT., 2016.

3. Lu, T.; Chen, F. Multiwfn: A Multifunctional Wavefunction Analyzer. J. Comput. Chem. 2012, 33, 580-92.

4. Humphrey, W.; Dalke, A.; Schulten, K. VMD: Visual Molecular Dynamics. J. Mol. Graph. 1996, $14,33-38$.

5. Manzhos, S.; Komatsu, M.; Nakazaki, J.; Segawa, H.; Yamashita, K. Theoretical Analysis of the Solvatochromism of Organic Dyes Differing by the Conjugation Sequence. J. Photonics. Energy. 2012, 2, No. 028001. 